\title{
Identification of candidate genes encoding the core components of the cell death machinery in the Ciona intestinalis genome
}

\author{
Dedicated to the memory of Mr Daichi Terajima
}

Cell Death and Differentiation (2003) 10, 749-753. doi: 10.1038/sj.cdd.4401223

Dear Editor,

Programmed cell death or apoptosis is a cell suicide mechanism that plays an essential role in the normal development and maintenance of tissue homeostasis in all multicellular organisms. ${ }^{1}$ Although many of the central participants of cell death signaling are conserved during evolution, the growth in the number of apoptotic proteins identified in the human genome indicates the complexity of programmed cell death in vertebrates. ${ }^{2}$ Chordates and vertebrates constitute the phylum chordata, and ascidians or sea squirts belong to urochordates. Ascidian has the smallest genome ( $\sim 16000$ protein coding genes) among all genetically manipulable chordate animals. ${ }^{3}$ Larva of ascidians possesses the basic developmental and morphological features of vertebrates including a prominent notochord and a dorsal central nervous system. ${ }^{4}$ Programmed cell death apparently takes place in these ascidians. ${ }^{5,6}$ For example, the epidermal cells in the tail of ascidian tadpole larva rapidly undergo apoptosis during metamorphosis (Figure 1). Thus, a comparative analysis of apoptosis genes in ascidian might provide an insight into the origin and evolution of the programmed cell death system.

To this end, we have used BLAST and Domain-centered analysis to search the JGI Ciona genome database (http:// grolithe.jgi-psf.org) and the ascidian cDNA/EST sequences from Kyoto University (http://ghost.zool.kyoto-u.ac.jp/indexr1.html) for apoptosis-related genes. ${ }^{3}$ In this article, we briefly describe the Ciona's molecular components in the core pathways of apoptosis and compare them with their counterparts in human, fly, and nematode by phylogenetic analysis.

Apoptosis is triggered within cells by the activation of a family of cysteine proteases called caspases that specifically cleave their substrates at aspartic acid residues. Caspases are produced in cells as catalytically inactive zymogens, and become activated by proteolytic processing during apoptosis. At least 14 caspases have been identified in mammals compared to three in nematode and seven in fly. ${ }^{2}$ Caspases involved in apoptosis are generally categorized as either upstream initiator caspases such as caspase-2, -8, -9, and -10 or downstream effector caspases including caspase-3, -6, and $-7 .^{7}$ The zymogen forms of initiator caspases contain extended $\mathrm{N}$-terminal prodomains such as death effector domain (DED) found in caspase-8 and -10 or caspaseassociated recruitment domain (CARD) in caspase-2 and -9 . These DED and CARD prodomains are important for their association with adapter molecules to trigger proteolytic autoactivation of the initiator caspases. In contrast, the effector caspases have short $\mathrm{N}$-terminal prodomains and their proteolytic processing and activation are largely dependent on upstream caspases.

We have identified 11 caspase genes in the $C$. intestinalis genome, of which four correspond to the initiator caspases containing either CARD or DED domain and seven belong to the effector caspases (Table 1). Of note, the CARD-containing caspases exist across species, while the DED-containing caspases are absent in nematodes. ${ }^{2}$ Therefore, the CARDcontaining caspases may share a common ancestry, whereas the DED-containing caspases may be specific to chordate animals with an immune system.

The phylogenetic analysis indicates that caspases are apparently separated into three major groups (Figure 2a). The first group consists of CARD-containing caspases, including the nematode caspase CED-3, Drosophila caspase DRONC, and human caspase-2. Two ascidian CARD-containing caspase genes (grail.37.27.1 and grail.48.81.1) belong to this
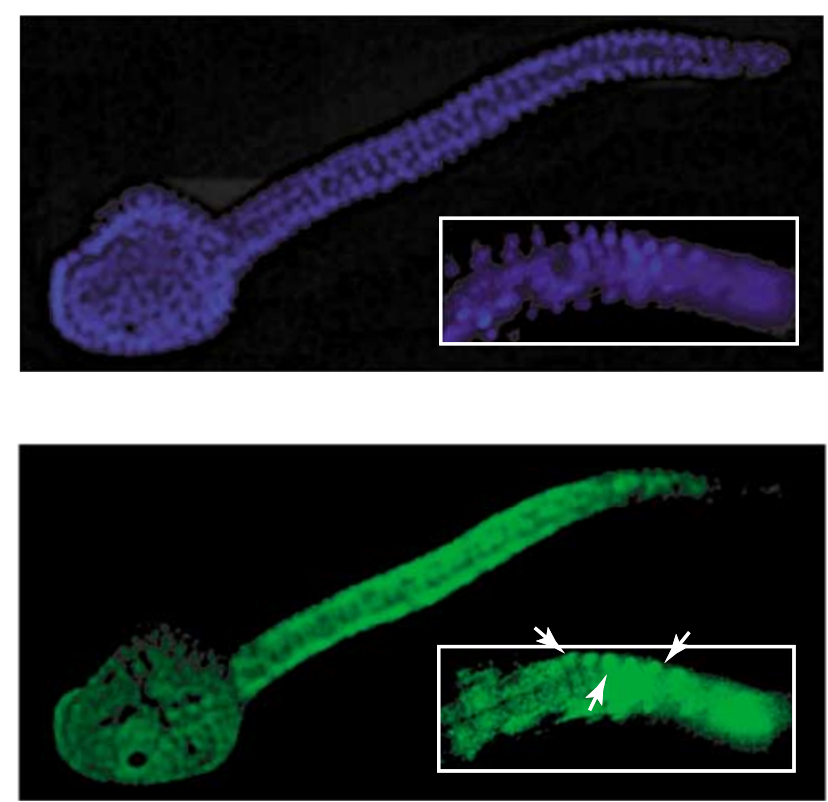

Figure 1 Epithelial cells in the tail of $C$. intestinalis tadpole die by apoptosis during tail morphogenesis. The nuclear morphology was examined by DAPI staining (top) and the apoptotic cells were detected by TUNEL assay (bottom) 
Table 1 Apoptosis-related genes in Ciona intestinalis

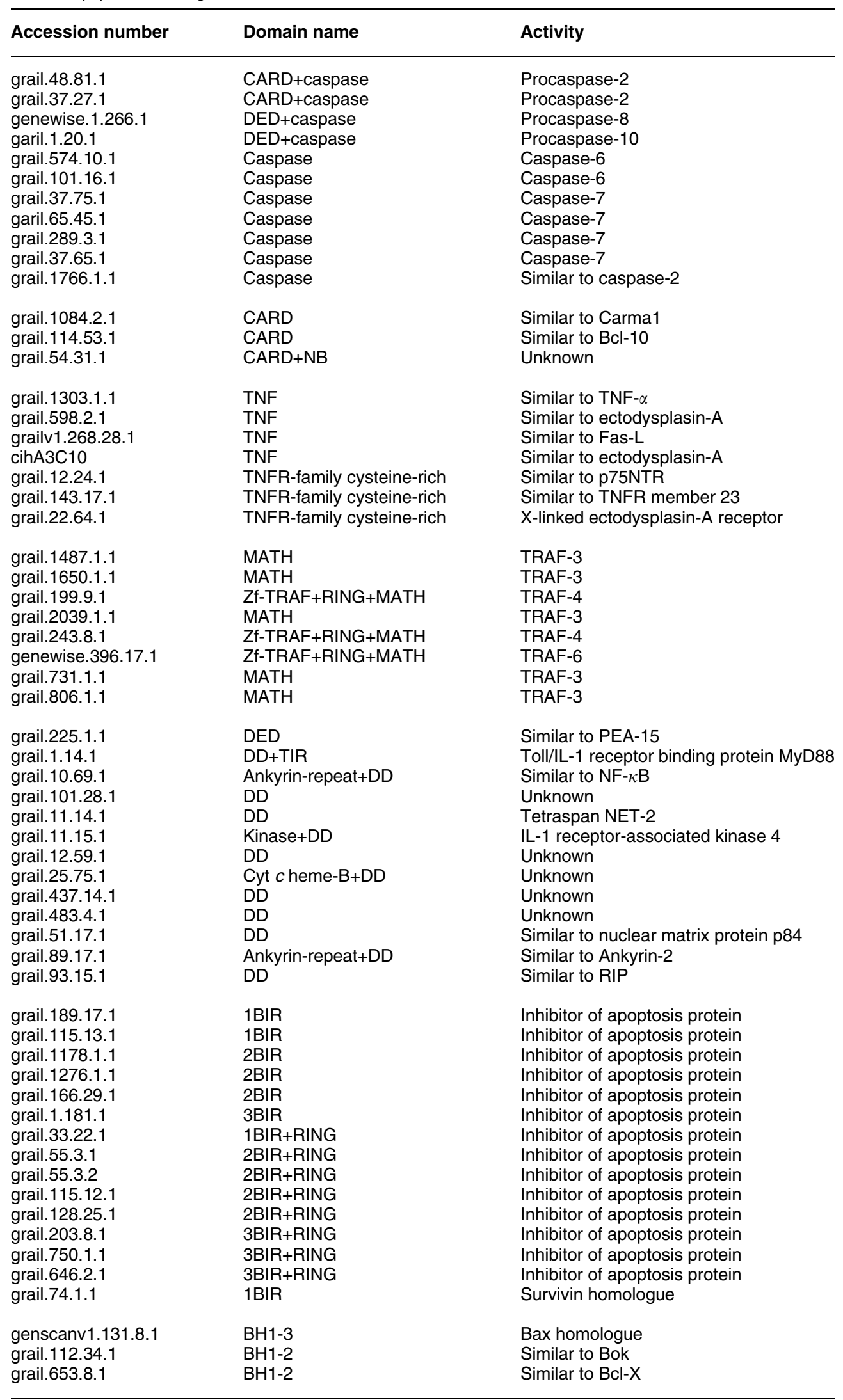




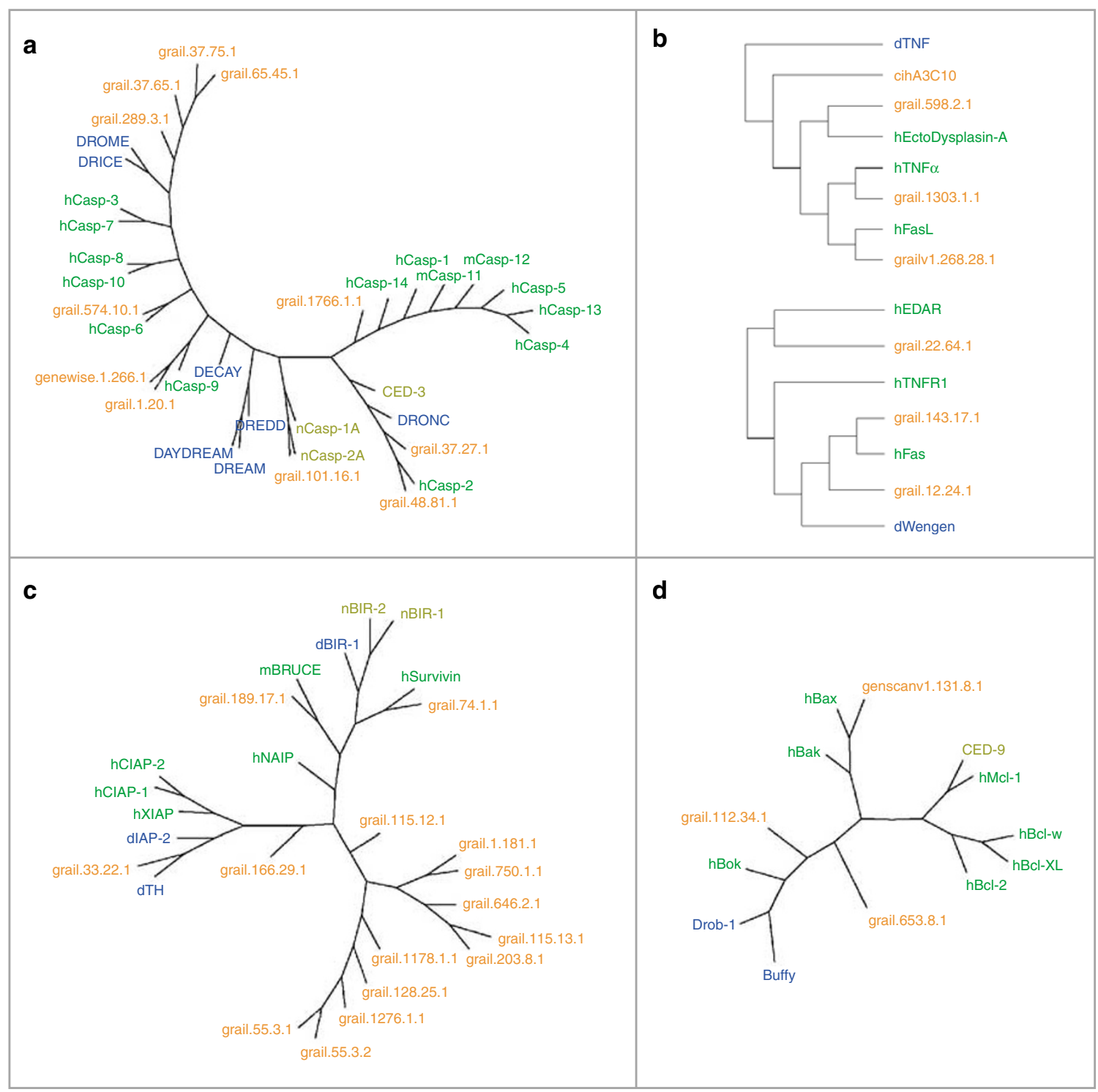

Figure 2 Phylogenetic trees of caspases (a), TNF (b), IAPs (c), and Bcl-2 family (d). Tree was based on alignments of complete sequences by the program CLUSTALX, followed by Phylip's PROTDIST and NEIGHBOR. Mammalian proteins are shown in green, Ciona in orange, Drosophila in blue, and C. elegans in gold

group, implying the evolution of this group of caspases from a common ancestry across the animal kingdom. Notably, the ascidian caspases are closer to human caspase-2 than DRONC and CED-3. Caspase-2 plays a pivotal role in DNA damage-induced cell death, ${ }^{8}$ an essential mechanism to maintain the genomic integrity in human and probably ascidians, whereas CED-3 and DRONC are key caspases controlling developmental cell death in Caenorhabditis elegans ${ }^{9}$ and Drosophila, ${ }^{10}$ respectively.

The second group contains a diversity set of caspases, including the human DED-containing initiator caspases (caspase-8 and -10) and effector caspases (caspase-3, -6, and -7 ) as well as the well characterized CARD-containing initiator caspase-9. Two nematode caspase-related proteins (Casp-1A and Casp-2A) and six Drosophila caspases including the only DED-containing fly caspase DREDD belong to this subfamily of caspases. Eight Ciona caspases including two DED-containing caspases (grail.1.20.1 and genewise.1.266.1) fall into this group. It has been well characterized that the initiator caspases (caspase-8 and -9) in this subfamily require the existence of downstream effector caspases to achieve apoptosis in response to intrinsic and/ or extrinsic death signals. This may be a reason why a diverse set of caspases such as DED-containing, CARD-containing, and effector caspases are found in this group. Particularly, in the case of ascidian, effector caspases (grail.289.3.1, 37.65.1, 37.75.1, and 65.45.1) show an intra-species expansion, indicating that they may have unique substrate specificities.

The third group of caspases includes human caspase-1, -4, $-5,-11,-12,-13$, and -14 , which are implicated in inflammatory response to infection, such as the processing and secretion of 
certain interleukins, rather than apoptosis. There is only one Ciona gene (grail.1766.1.1) related to this subfamily of caspases but counterparts are found neither in $C$. elegans nor in Drosophila, suggesting that this group of caspases evolved later and may be specific to chordate animals. In Drosophila, however, the inflammatory caspase is found in the second group. DREDD has been reported to play an important role not only in apoptosis but also in antibacterial response ${ }^{11}$ and activation of Relish, ${ }^{12}$ a member of the NF- $\kappa \mathrm{B}$ family of transcription factors.

Genetic studies have provided convincing evidence for the existence of an intrinsic pathway for caspase activation in $C$. elegans. ${ }^{9}$ The nematode caspase CED-3 is activated by its interaction with the adapter protein CED-4 in cells fated to die. ${ }^{13}$ CED-4 contains a CARD domain and a nucleotidebinding (NB) oligomerization domain. The CARD domain in CED-4 binds to homologous structure within the prodomain of CED-3. Interaction with CED-4 seems to be the only mechanism for CED-3 activation, because there is no apoptosis in ced-4 mutant worms. There is a Drosophila CED-4 homolog (DARK), which is important for cell death in flies. ${ }^{14}$ Apaf- 1 is the only CED-4 homolog found so far in mammals. Unlike CED-4 and DARK, however, the release of cytochrome $c$ from mitochondria is required for Apaf-1 to trigger the activation of the initiator caspase-9. ${ }^{15}$ The $C$. intestinalis genome contains three genes encoding CARDcontaining adapter-like proteins (Table 1). Two of these genes contain only CARD as a functional domain. The grail.114.53.1 shares a sequence similarity with $\mathrm{Bcl}-10$ and the grail.1084.2.1 with the $\mathrm{Bcl}-10$-binding protein Carma1. Both Bcl-10 and Carma1 are implicated in activation of NF- $\kappa \mathrm{B}^{16}$ rather than in caspase activation. The other gene (grail.54.31.1) in Ciona contains a CARD and a putative NB domain both of that commonly found in the CED-4/Apaf-1related proteins. However, the phylogenetic analysis shows that there are no significant similarities between grail.54.31.1 and CED-4/Apaf-1 genes (data not shown). Thus, it requires experimental evidence to prove whether there are functional homologs of CED-4/Apaf-1 in ascidians.

In contrast to nematode, mammals also utilize death receptors such as TNFR1, Fas, DR3, DR4, or DR5 for the activation of caspases. Binding of ligand to these receptors on the cell surface recruits adapter proteins such as FADD to the cytoplasmic DD of the receptors. FADD, in turn, recruits the initiator caspase-8 through interactions between the DED domains of these proteins to form a death-inducing signaling complex (DISC) that triggers caspase-8 activation. ${ }^{17}$ As can be seen in Table 1, four putative members of the TNF family such as TNF- $\alpha$ like (grail.1303.1.1), ectodysplasin-A like (grail.598.2.1 and cihA3C10), and Fas-L like (grailv1.268.28.1) along with three possible TNFR-related genes (grail.12.24.1, 143.17.1, and 22.64.1) have been detected in the $C$. intestinalis genome. The phylogenetic analysis in Figure 2B indicates that each member of the TNF and TNFR families in human has more sequence similarities to their corresponding homologs in Ciona compared to Drosophila.

The TNFR-associated factors (TRAFs) are the major signal transducers for the TNFR superfamily. All members of the TRAF family share a conserved region termed meprin- and TRAF-homology (MATH) domain that is important for their dimerization. Six members of the TRAF family have been identified in mammals, which are thought to be important regulators of not only cell death but also immunity. ${ }^{18}$ Ciona contains eight TRAF-related genes with high sequence similarities to TRAF-3, -4 , or -6 (Table 1 ). The number of TRAF genes in Ciona is comparable to that in human and considerably higher than that in Drosophila and C. elegans ( 3 and 1 , respectively). ${ }^{2}$ This is consistent with the fact that ascidians are much closer to humans than to flies from the evolution standpoint. Although there is no evidence for the existence of adaptive immunity in ascidians, Ciona clearly contains numerous genes related to innate immunity. ${ }^{3}$ Detection of eight TRAF-related genes in Ciona is in accordance with the evidence that many molecular components involved in apoptosis overlap with those involved in the immune responses to infection. ${ }^{19}$

In addition to CARD, the DED and DD domains are other classes of apoptotic adapters. As shown in Table 1, the $C$. intestinalis genome contains one DED adapter protein related to PEA-15 and 12 DD-containing genes including those homologous to MyD88, NF- $\kappa \mathrm{B}$, NET-2, IL-1 receptor-associated kinase 4, nuclear matrix protein p84, Ankyrin-2, and RIP, although a FADD homolog is not detected. The number of DED- and/or DD-containing adapter proteins is lower than that in vertebrates but higher than that in nematode and fly. ${ }^{2}$

The inhibitor of apoptosis (IAP) family of proteins represents an important class of endogenous inhibitors of caspases. $^{20}$ IAPs bind to and potently inhibit the initiator caspase, caspase- 9 , and the effector caspases, caspase-3 and -7. Eight distinct IAPs, including XIAP, c-IAP1, c-IAP2, and survivin in mammals, four in Drosophila, and two in $C$. elegans have been identified to date. ${ }^{2}$ All IAPs contain at least one baculovirus IAP repeat (BIR) domain. XIAP, c-IAP1, and c-IAP2 contain three BIR domains each, in which the third BIR domain (BIR3) inhibits the enzymatic activity of activated caspase-9, whereas the linker region between the first BIR domain (BIR1) and the second BIR domain (BIR2) selectively targets caspase- 3 and $-7 .{ }^{21}$ Some IAPs also contain a ring finger located near the C-terminus of these proteins, which is implicated in ubiquitin-proteasome-mediated protein degradation. ${ }^{22}$ The $C$. intestinalis genome contains 15 BIR-containing proteins, of which eight contain both BIR and ring finger domains (Table 1). The phylogenetic analysis in Figure $2 \mathrm{c}$ indicates that two Ciona genes (grail.33.22.1 and grail.166.29.1) are present in the same branch with XIAP, a prominent inhibitor of caspases, although they are not close to each other. In contrast, the human survivin gene, which is involved in mitosis rather than apoptosis regulation, is located in a second branch and is highly conserved in Ciona as well (see grail.74.1.1). Interestingly, ascidian has a speciesspecific expansion of many IAP-related genes as evident as a third branch. This may reflect an ecological and physiological relevance of these BIR-containing proteins that are uniquely important to sea squirts.

The Bcl-2 family proteins are the central regulators of programmed cell death and apoptosis by controlling the activation of caspases. Both cell death inhibitors and promoters exist in this protein family, and the relative ratios of pro- and antiapoptotic Bcl-2 family proteins arbitrate the life or death decision. The antiapoptotic proteins such as Bcl-2 
and $\mathrm{Bcl}-\mathrm{XL}$ tend to prevent the release of apoptogenic molecules such as cytochrome $c$ from mitochondria and subsequent caspase activation, whereas the proapoptotic proteins such as Bax and Bok promote these events. ${ }^{23}$ All members of this family possess at least one of the four conserved motifs known as $\mathrm{Bcl}-2$ homology domains: $\mathrm{BH} 1$, $\mathrm{BH} 2, \mathrm{BH} 3$, and $\mathrm{BH} 4 .^{24}$ The antiapoptotic members of the Bcl2 family share all the conserved $\mathrm{BH}$ domains $(\mathrm{BH} 1-4)$ that are important for their antiapoptotic activity. The proapoptotic $\mathrm{Bcl}-$ 2 family proteins are further divided into the $\mathrm{BH} 1-3$ multidomain (Bax, Bak, and Bok) and BH3-only (Bad, Bim, and EGL-1) subfamilies.

More than a dozen Bcl-2 family proteins have been reported in mammals. In contrast, only two Bcl-2 family members with opposing functions, termed as CED-9 and EGL-1, have been identified in $C$. elegans. CED-9 has all $\mathrm{BH} 1-4$ domains, is a functional homologue of $\mathrm{Bcl}-2$, and inhibits the activation of the nematode caspase CED-3. On the other hand, EGL-1 is an BH3-only proapoptotic protein and triggers CED-3 activation by antagonizing the CED-9 function. ${ }^{9}$ However, the Bax family proapoptotic proteins containing $\mathrm{BH} 1-3$ are apparently absent in worms. In contrast, Drosophila has two genes related to the $\mathrm{BH} 1-3$ proapoptotic protein Bok, but so far neither counterpart of the antiapoptotic nor of BH3-only proapoptotic Bcl-2 family genes have been reported in flies. As seen in Figure $2 d$, the Ciona genome contains three genes related to the $\mathrm{Bcl}-2$ family proteins, of which one is the homolog of human Bax and other two are clustered together with human Bok and its Drosophila homologues. Interestingly, Bok is segregated from Bax and Bak on the phylogenetic tree, although these three are thought to belong to the same subfamily of the Bcl-2 family proteins. Moreover, the grail.653.8.1 encodes a putative protein with a strong sequence similarity to human $\mathrm{Bcl}-\mathrm{XL}$ (Table 1 ), but it is not present on the same branch with Bcl-XL in the phylogenetic tree. This suggests that the grail.653.8.1 could be an intermediate of evolution of the $\mathrm{BH}$ domains commonly found in the anti- versus proapoptotic Bcl-2 family proteins. In addition, the $\mathrm{BH} 3$-only genes have been identified in various vertebrates but not in invertebrates except the nematode $C$. elegans. We also failed to identify any apparent $\mathrm{BH} 3$-only $\mathrm{Bcl}-$ 2 family members in the $C$. intestinalis genome. It could be because of a lack of any statistically significant motif in the $\mathrm{BH}$-only proapoptotic proteins that allows reliable sequencebased predictions.

Taken together, our results clearly indicate that ascidians and vertebrate animals, when compared to worms and flies, share many central participants of the cell death signaling machinery. This may not be surprising, considering that programmed cell death is a highly conserved on one hand but, on the other hand, a complicated process characteristically involved in development, stress and inflammatory responses, aging, and diseases of animals. Thus, the functional domains in the main players of apoptosis maybe evolved from the common ancestry, but they show diverse expansion after speciation. The results in the present study provide a basis to further study the origin and evolution of programmed cell death mechanisms. In addition to their unique evolutionary position, the simplicity of cellular and genomic architecture of ascidians could provide an excellent genetic tool to investigate the molecular mechanisms of apoptosis during development. Also, there is a potential to use ascidians and their genes to probe molecular targets of genetic diseases as well as to screen therapeutic drugs.

\section{$D$ Terajima ${ }^{1}, K$ Shida $^{2}, N$ Takada $^{3}, A$ Kasuya ${ }^{2}$, D Rokhsar ${ }^{4}, \mathrm{~N} \mathrm{Satoh}^{5}, \mathrm{M} \mathrm{Satake}^{1}$ and H-G Wang ${ }^{\star, 2,3}$}

1 Department of Molecular Immunology, Institute of Development, Aging and Cancer, Tohoku University, Seiryo-machi, Aoba-ku, Sendai 980-8575, Japan

2 Center for Interdisciplinary Research, Tohoku University, Aramaki Aza Aoba, Aoba-ku, Sendai 980-8578, Japan

${ }^{3}$ Drug Discovery Program, H Lee Moffitt Cancer Center \& Research Institute, 12902 Magnolia Drive, Tampa, Florida 33612, USA

${ }^{4}$ US Department of Energy Joint Genome Institute, 2800 Mitchell Drive, Walnut Creek, CA 94598, USA

${ }^{5}$ Department of Zoology, Graduate School of Science, Kyoto University, Kyoto 606-8502, Japan

* Corresponding author: Hong-Gang Wang, Drug Discovery Program, Moffitt Cancer Center, 12902 Magnolia Drive, Tampa, FL 33612, USA; Phone: 813979-9764; Fax: 813-979-6748; E-mail: wanghg@moffitt.usf.edu

1. Raff MC (1992) Nature 356: $397-400$

2. Aravind L et al. (2001) Science 291: $1279-1284$

3. Dehal $P$ et al. (2002) Science 298: 2157-2167

4. Corbo JC et al. (2001) Cell 106: 535-538

5. Jeffery W (2002) Mech. Dev. 118: 111-124

6. Chambon JP et al. (2002) Development 129: 3105-3114

7. Salvesen GS and Dixit VM (1997) Cell 91: 443-446

8. Lassus $\mathrm{P}$ et al. (2002) Science 297: 1352-1354

9. Horvitz HR (1999) Cancer Res. 59: 1701s-1706s

10. Kumar S and Doumanis J (2000) Cell Death Differ. 7: 1039-1044

11. Leulier $\mathrm{F}$ et al. (2000) EMBO Rep. 1: 353-358

12. Stoven $S$ et al. (200) EMBO Rep. 1: $347-352$

13. Yang $X$ et al. (1998) Science 281: 1355-1357

14. White K (2000) Curr. Biol. 10: R167-R169

15. Wang X (2001) Genes Dev. 15: 2922-2933

16. Gaide $O$ et al. (2001) FEBS Lett. 496: $121-127$

17. Ashkenazi A and Dixit VM (1988) Science 281: 1305-1308

18. Chung JY et al. (2002) J. Cell Sci. 115: 679-688

19. James ER and Green DR (2002) Cell Death Differ. 9: 355-357

20. Deveraux QL and Reed JC (1999) Genes Dev. 13: 239-252

21. Shi $Y$ (2002) Mol. Cell 9: $459-470$

22. Martin SJ (2002) Cell 109: 793-796

23. Green DR and Reed JC (1998) Science 281: 1309- 1312

24. Reed JC (1998) Oncogene 17: 3225-3236 